\title{
A study on the unphysical mass transfer of SCMP pseudopotential LBM
}

\author{
Wei Gong ${ }^{1}$, Yuying Yan ${ }^{*} 1,2$, Sheng Chen $^{1}$ \\ ${ }^{1}$ Fluids \& Thermal Engineering Group, Faculty of Engineering, University of \\ Nottingham, University Park, Nottingham, United Kingdom \\ ${ }^{2}$ Center for Fluids \& Thermal Engineering, University of Nottingham Ningbo, China \\ *corresponding author: yuying.yan@nottingham.ac.uk
}

\begin{abstract}
In general, a multi-bubble/droplet configuration cannot sustain a steady state using singlecomponent multiphase (SCMP) pseudopotential lattice Boltzmann method (LBM). Our study shows that the unachievable multi-bubble/droplet system is due to an unphysical mass transfer, which we call it "the big eat the small" - the smaller bubbles/droplets become smaller shrink, and eventually disappear while the bigger ones get bigger and bigger without a physical coalescence. In our present study, the unphysical mass transfer phenomenon is investigated, and the possible reason is explored. It is found that there is a spurious flow field formed between two bubbles or droplets with different shapes, and such flow field is exactly the transfer of high-density mass. In addition, it is found that the curvatures of the interfaces determine the direction of the spurious flow field, and for the definition of "the big eat the small", "the big" refers to the interfaces that have larger radii of curvature while "the small" represents the interfaces with smaller radii of curvature. Multi-component multiphase (MCMP) LBM is also tested in this work and it is found to be free of the unphysical mass transfer. Moreover, all the cases show by analysing the unphysical mass transfer phenemena it can be summarized that the most likely reason for of the unphysical mass transfer might be the essential attractive interaction forces of the pseudopotential LBM.
\end{abstract}

Keywords: SCMP pseudopotential LBM, unphysical mass transfer, interface curvature

\section{Introduction}

In the past few decades, the lattice Boltzmann method (LBM) has been developed significantly due to its remarkable advantages compared with conventional CFD methods in mesoscale [1,2]. Meanwhile it has been applied to a broad range of application areas [3-9], such as single/multiphase flows, phase change, rarefied gas flows, etc. Among all the members of the LBM 
community including the colour-gradient LBM [10], the pseudopotential LBM [5], the freeenergy LBM [11] and the phase-field LBM [12,13], the pseudopotential model has shown striking superiority in multiphase flows due to its simplicity, versatility and the distinctive feature that different phases can be segregated automatically on account of the particle interactions. Besides, in comparison with the free-energy and the colour-gradient LBM, the pseudopotential LBM has much better performance in dynamic multiphase flows at large density ratios and relatively high Reynolds numbers [7].

The pseudopotential LBM was firstly introduced by Shan and Chen $[14,15]$, and the most distinctive feature of this model is the interparticle potential $\Psi$ which is based on the local density. With the pseudopotential treatment, the particles having the same densities can be attracted together, thus causing the phase segregation automatically without any special treatment of interface tracking or capturing techniques. The original definition of the pseudopotential proposed by Shan and Chen is $[14,15]$

$$
\Psi=\rho_{0}\left(1-e^{-\frac{\rho}{\rho_{0}}}\right)
$$

where $\rho$ denotes density and $\rho_{0}$ is a constant. The interaction force at the position $\mathbf{x}$ and time $t$ acting on a partible from the other surrounding particles for single-component multiphase (SCMP) models can be expressed as

$$
\boldsymbol{F}_{\text {int }}(\boldsymbol{x}, t)=-G \Psi(\boldsymbol{x}) \sum_{\alpha} w_{\alpha} \Psi\left(\boldsymbol{x}+\boldsymbol{e}_{\alpha} \delta_{t}\right) \boldsymbol{e}_{\alpha}
$$

where $G$ is a parameter that determines the strength of the interaction force, $w_{\alpha}$ are the weights used to calculate the isotropic interaction force, and $\mathbf{e}_{\alpha}$ and $\delta_{t}$ are the discrete lattice velocities and time step, respectively. With Taylor expansion to the interaction force equation (2), the following non-ideal equation of state (EOS) can be obtained [5]

$$
p=\rho c_{s}^{2}+\frac{G c_{s}^{2}}{2} \Psi^{2}
$$

with which other EOSs such as van der Waals (vdW) EOS [16], Carnahan-Starling (C-S) EOS [17] and Peng-Robinson (P-R) EOS [18] can be incorporated into the pseudopotential model by replacing the potential equation (1) with the following definition [19]

$$
\Psi=\sqrt{\frac{2\left(p-\rho c_{s}^{2}\right)}{G c_{s}^{2}}}
$$


Incorporating the realistic EOSs into the pseudopotential LBM is an important and effective way to increase the density ratio of simulation, which has always been a big issue restricting the application of LBM for real multiphase flows.

In recent years, advances for development of the pseudopotential LBM have been focusing on the spurious current [20], thermodynamic inconsistency [21], limited density and viscosity ratios [22] by adjusting the equation of state (EOS) [18], interaction force term [23] and force scheme [24]. Meanwhile, applications of pseudopotential LBM can be found in a wide range of areas such as boiling heat transfer [25], condensation [26], droplet motions [27] etc. Some excellent latest review works regarding pseudopotential LBM can be found in references $[2,5,7]$.

Our previous study shows that the multiple bubbles/droplets cannot coexist stably using the normal SCMP models [28], where only the attractive forces between particles are considered which drive the phase segregation. The reason for the unachievable multi-bubble/droplet system is the unphysical mass transfer which we call it "the big eat the small" - the smaller bubbles/droplets become smaller shrink and eventually disappear over time while the bigger ones get bigger before a physical coalescence. Chibbaro et al. [23] presented a new model with midrange repulsion forces introduced into the interaction forces by involving the second nearest-neighbouring particles. This repulsive force mimics the impurities on the liquid/vapour interface that prevents the coalescence of contacted droplets/bubbles to achieve multibubble/droplet coexistence as shown in Fig. 1. However, after testing their model, it is found that the unphysical phenomenon of "the big eat the small" still cannot be eliminated. As shown in Fig. 1(b) and Fig. 1(c), it can be seen that the smaller bubbles still get smaller over time. The reason that there are more small droplets left in Fig. 1(a) than bubbles in Fig. 1(c) at the same time level is that the unphysical phenomenon of mass transfer is much less severe for multidroplet configurations than the multi-bubble configurations.

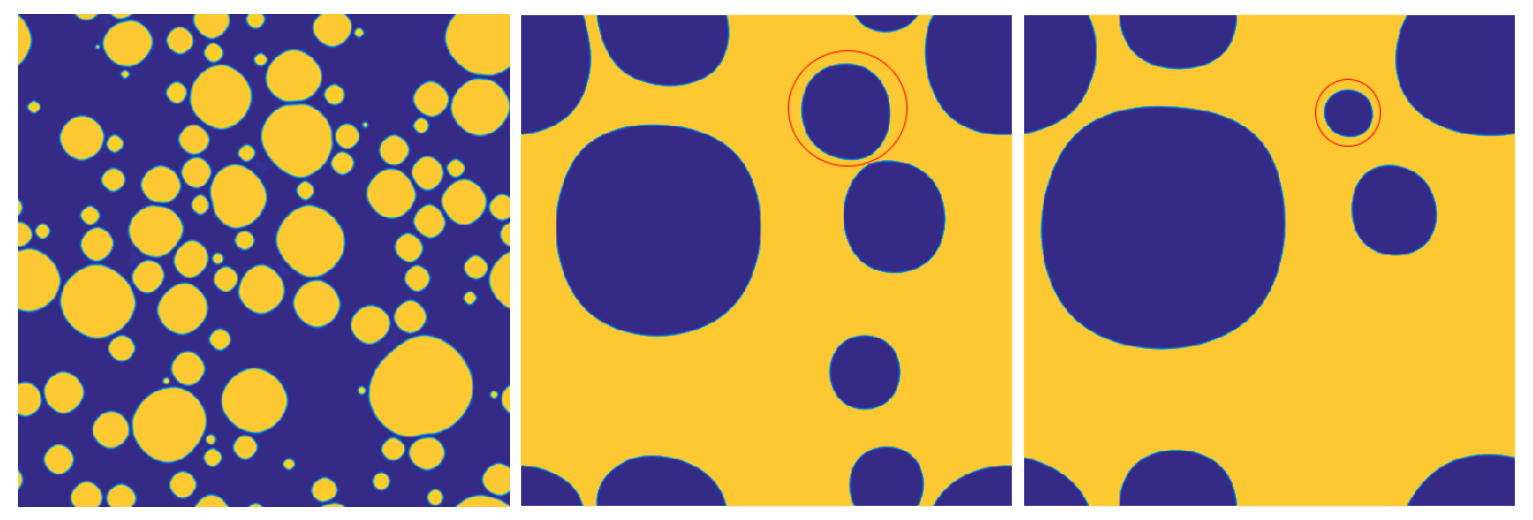


Fig. 1 Multi-bubble/droplet configurations in $512 \times 512$ computational domains with periodical boundary conditions using the midrange repulsion model (a) multi-droplet at $t=$ $20,000 \delta_{t}$ (b) multi-bubble at $t=15,000 \delta_{t}$ (c) multi-bubble at $t=20,000 \delta_{t}$

Besides the use of SCMP models, multi-component multiphase (MCMP) models are also widely applied, and the interaction force for this model can be written as [14]

$$
\boldsymbol{F}_{i n t, \sigma}(\boldsymbol{x}, t)=-\Psi_{\sigma}(\boldsymbol{x}) \sum_{\bar{\sigma}} G_{\sigma \bar{\sigma}} \sum_{\alpha} w_{\alpha} \Psi_{\bar{\sigma}}\left(\boldsymbol{x}+\boldsymbol{e}_{\alpha} \delta_{t}\right) \boldsymbol{e}_{\alpha}
$$

where $\sigma$ and $\bar{\sigma}$ denote the corresponding components. Using MCMP models to study multiphase flows involving multi-bubble/droplet systems is more popular than SCMP models, and such studies can be found in reference [29-35]. However, the MCMP always suffers the limitation of increasing the density ratio and kinematic viscosity ratio [5], which restricts its application to a large extent.

Our previous paper [28] pointed out the problem of "the big eat the small" in SCMP model for the first time and its behaviours in thermal multiphase flow was presented, where we found by coupling an entropy-based energy equation the unphysical mass transfer can be effectively restrained. In this paper, the features of the unphysical mass transfer phenomenon are studied in detail and the possible reason from the viewpoint of algorithm is discussed, to have a better understanding of this problem and lay the foundation for completely sorting it out in further studies.

\section{Numerical simulation and discussion}

The original Shan-Chen model [14], an improved Bhatnagar-Gross-Krook (BGK) model in 3D with the given code in reference [36], the Gong-Cheng model [37], the midrange repulsion model [23] and the Li Q. improved multiple-relaxation-time (MRT) model [38] are all tested in our study. These models contain the typical non-ideal EOSs, forcing schemes, and collision terms operators of SCMP pseudopotential LBM. After testing these models, it is found that none of them can get rid of the unphysical mass transfer. In this paper the Li Q. model [38] is used to present the study of the unphysical mass transfer features.

With the realistic non-ideal EOS applied in the SCMP model, this model is verified by comparing the simulation result with the Maxwell construction

$$
\int_{V_{m, l}}^{V_{m, v}} p_{E O S} d V_{m}=p_{0}\left(V_{m, v}-V_{m, l}\right)
$$


where $V_{m}$ is the molar volume, and the subscript $v$ and $l$ denote vapour and liquid respectively. When the saturation temperature $T_{s}=0.86 T_{c}$, the liquid and vapour densities are $\rho_{l} \approx 0.65$ and $\rho_{v} \approx 0.38$ in the two-phase system, which correspond to the coexistence densities calculated by Eq. (6). Other fluids properties as set as follows. The kinetic viscosities for liquid and vapour phases are $v_{l}=0.1$ and $v_{v}=0.5 / 3$. The specific heat is taken as constant $c_{v}=6$. The thermal conductivity is $\lambda=\rho c_{v} \chi$ with $c_{v} \chi=0.028$. For the MCMP simulation, the original Shan-Chen model [14] is applied, where no non-ideal EOS is considered, therefore the model is verified with the Laplace Law

$$
\Delta p=\frac{2 \sigma^{*}}{r}
$$

where $\sigma^{*}$ is the surface tension. The verification can be seen in Fig. 2.

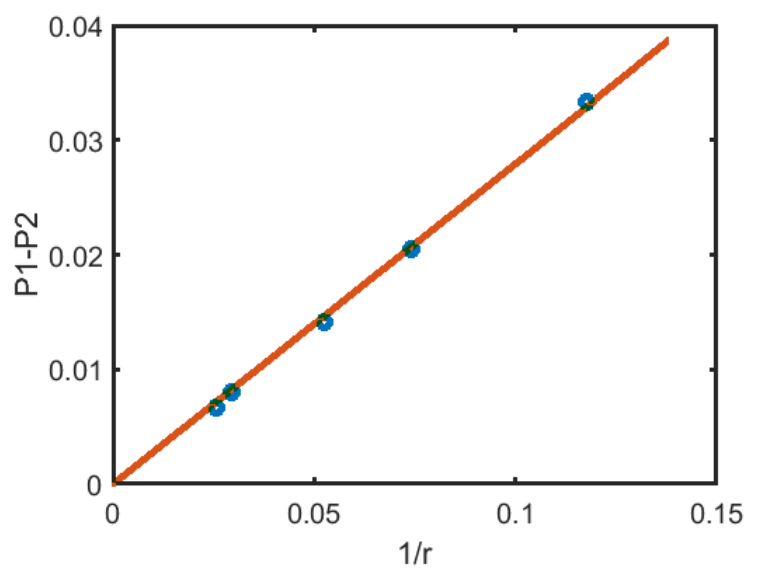

Fig. 2 Verification of Laplace Law for the MCMP model

Why the unphysical mass transfer just occurs in multi-bubble/droplet systems as the process of "the big eat the small"? Is there "the small eat the big"? In this part a specific particular twophase system as shown in Fig. 3 is tested in a $100 \times 150$ computational area. The top and bottom boundaries are solid walls and the left and right boundaries are periodical. The initial states are shown in Fig. 3(a) and Fig. 3(e), where the initial radium of the bubble/droplet is 40, the height of the bottom vapour/liquid region is 10 , and no initial velocity is loaded. In such an initial state, either the volume or the area of the liquid/vapour interface of the bottom vapour/liquid region is smaller than that of the bubble/droplet. However, this time it is the small that "eat" the big, rather than the normal phenomenon "the big eat the small". Therefore adding the unphysical problem in the regular bubble/droplet systems it can be concluded that it is the bubbles/droplets having bigger interface curvature radii that absorb the ones with smaller 
interface curvature radii, rather than the volume of the bubble/droplet or the area of the interfaces.

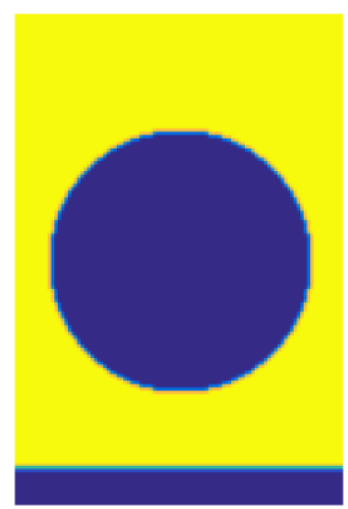

(a)

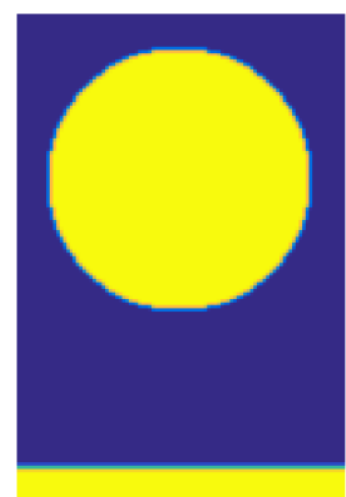

(e)

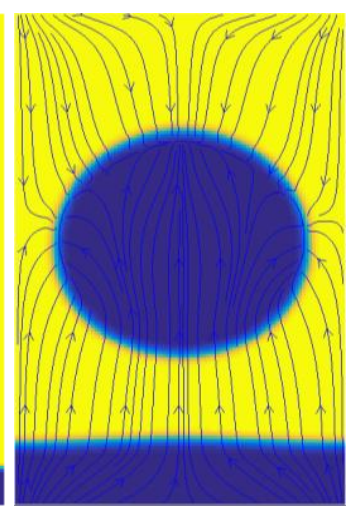

(b)

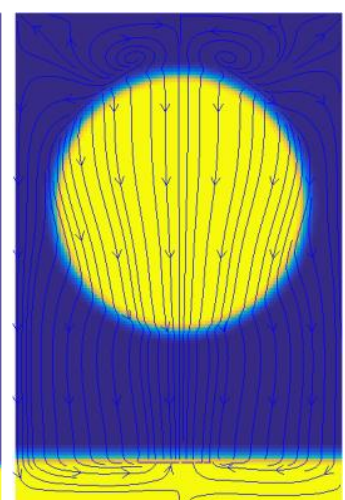

(f)

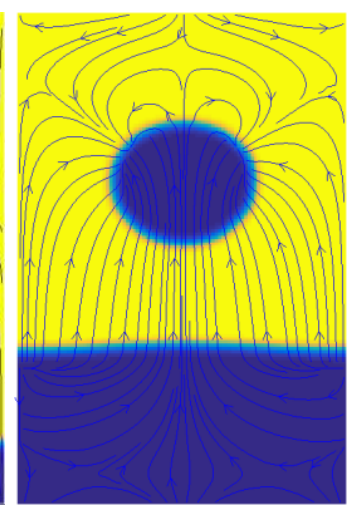

(c)

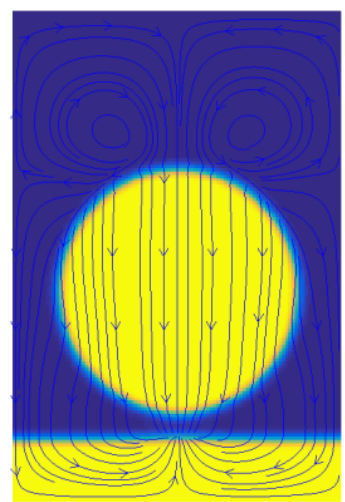

$(\mathrm{g})$

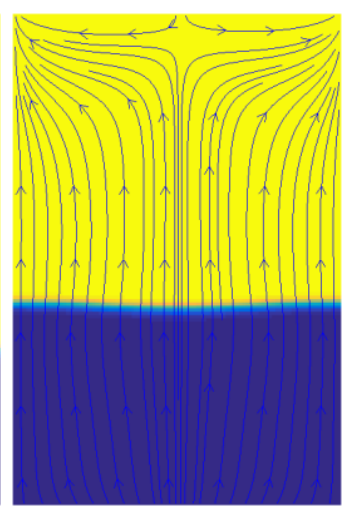

(d)

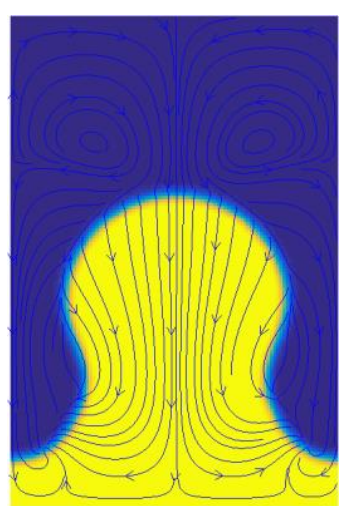

(h)

Fig. 3 The small vapour/liquid region "eats" the big bubble/droplet (a) bubble system, $t=$ 0 (b) bubble system, $t=1,100 \delta_{t}$ (c) bubble system, $t=2,500 \delta_{t}$ (d) bubble system, $t=$ $3,700 \delta_{t}$ (e) droplet system, $t=0$ (f) droplet system, $t=4,200 \delta_{t}$ (g) droplet system, $t=$

$$
9,400 \delta_{t}(\mathrm{~h}) \text { droplet system, } t=10,200 \delta_{t}
$$

From Fig. 3 it can be also seen that spurious flow fields are generated between bubbles/droplets. Moreover, the direction of the spurious flow field is exactly the direction of the high-density mass transfer, which means the unphysical mass transfer phenomenon is the transfer of highdensity mass. Besides in the normal SCMP pseudopotential LBM only attractive interaction force is considered, and this interaction force only acts on the area where there is potential gradient, and the interaction force has the same direction as with the potential gradient, which can be concluded from Eq. (2). Normally the high-density particles have high pseudopotentials, thus it can be in a manner of speaking that the essence of the normal SCMP pseudopotential LBM is the mutual attraction of high-density particles. Adding that the unphysical transfer is 
related to the curvatures of the two-phase interfaces, and the magnitude of the interaction forces are apparently concerned with the interface curvature - smaller curvature generates larger interaction force, it is most likely that the reason for of the unphysical mass transfer is the essential attractive interaction force. To confirm this viewpoint about the reason for ef the unphysical mass transfer, another example is given in Fig. 4, where an annular liquid film is initially placed in a static state. It can be seen that eventually the annular liquid film is agglomerated to a sphere liquid droplet, which is more evident to suggest show the high-density mass moving driven by the attraction force.

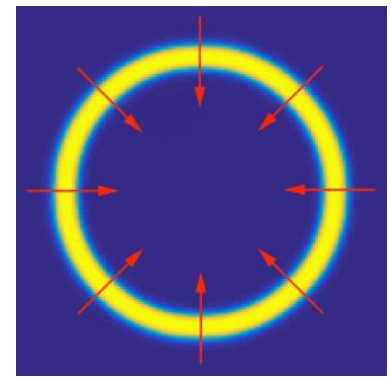

(a)

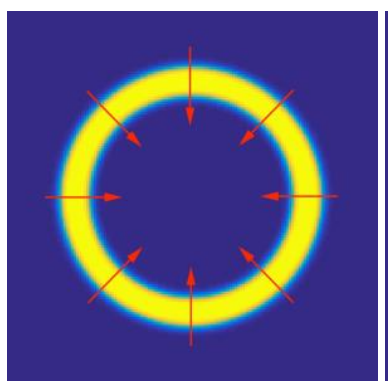

(b)

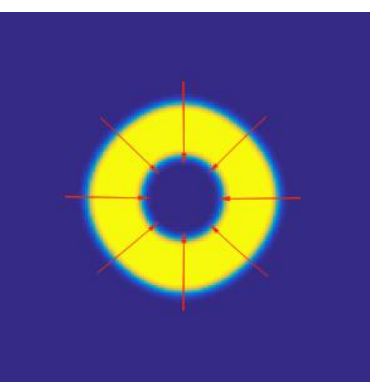

(c)

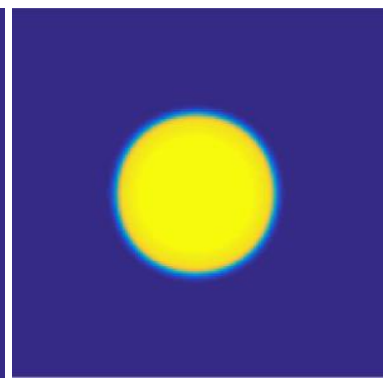

(d)

Fig. 4 The evolution of an annular liquid film (a) $t=41 \delta_{t}$ (b) $t=521 \delta_{t}$ (c) $t=1,001 \delta_{t}$ (d) $t=1,361 \delta_{t}$

Fig. 5 presents the evolution of multi-bubble/droplet system using MCMP model, and there is no unphysical mass transfer observed over time. Fig. 6 gives the comparison of densities and spurious currents of the two different components. From Fig. 6(c) and Fig. 6(d), it can be seen that the spurious currents point to opposite directions due to the density distributions of the two components. Apparently the spurious currents in this model are mainly caused by the attractive interaction forces, and the attractive forces of the two components point to the opposite ways, thus any unphysical effect owing to the attractive interaction force can be counteracted, which should be the reason that there is no such unphysical mass transfer in MCMP LBM.
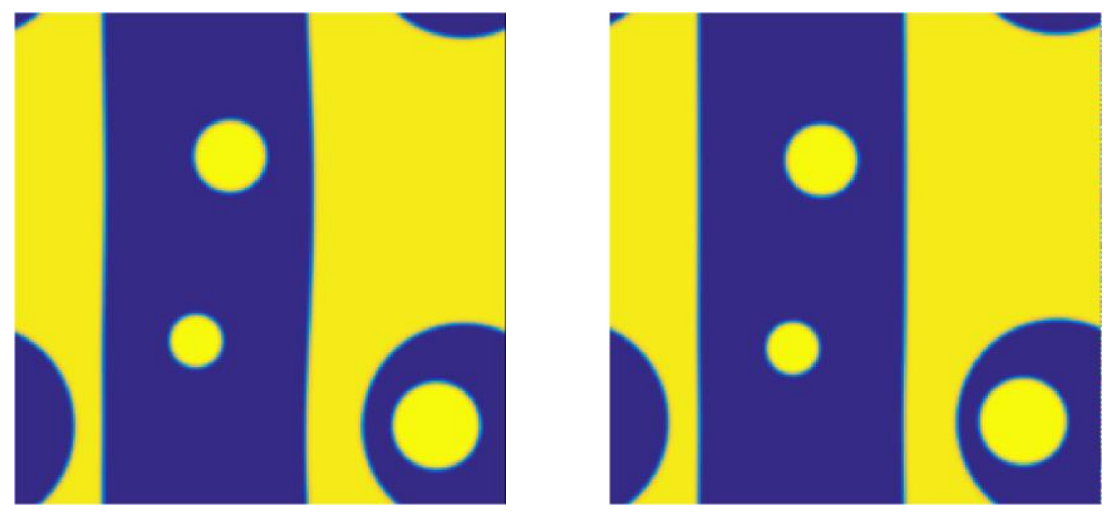
Fig. 5 Multi-bubble/droplet system evolution using MCMP model in a $200 \times 200$ area (a) $t=15,000 \delta_{t}$ (b) $t=20,000 \delta_{t}$

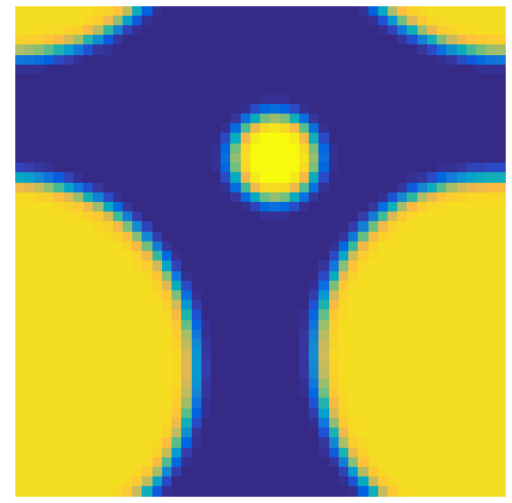

(a)

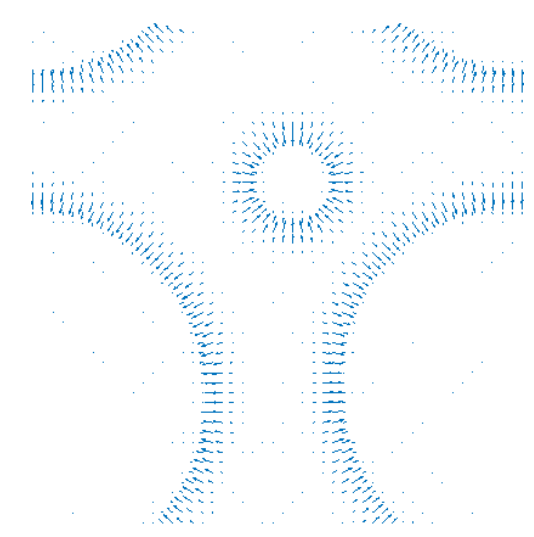

(c)

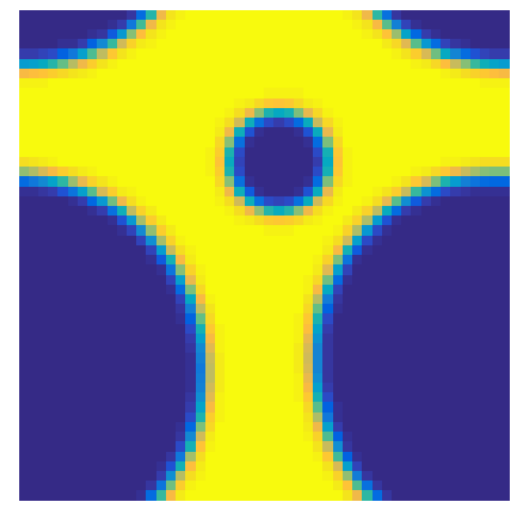

(b)

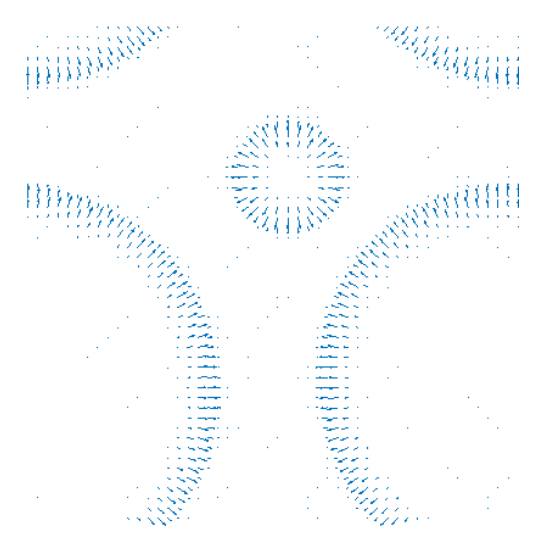

(d)

Fig. 6 Comparison of the two components of MCMP model in a $50 \times 50$ area at $t=$ $2,000 \delta_{t}$ 
Interestingly, for multi-droplet the unphysical mass transfer seems to be similar to the Ostwald ripening, which has been observed in solid solutions or liquid sols describing the change of an inhomogeneous structure over time that, small crystals or sol particles dissolve and redeposit onto larger crystals or sol particles [39]. The reason for Ostwald ripening is that larger particles are more energetically favoured than smaller particles, because molecules on the surface of a particle are energetically less stable than the ones in the interior and smaller particles have larger specific area [40]. Therefore, Ostwald ripening is related to the free energy at the level of molecular scale and particles motion. However in pseudopotential model the so called "particle" is density distribution function, not real particles and the mechanism for multi-phase flow are gas kinetic theory Boltzmann equation and fluid mechanics Navior-Stokes equations. That is also the reason we call problem "unphysical", because in the general immiscible twophase systems which can be described by Navior-Stokes equation there should not be such phenomenon.

\section{Conclusions}

In the present study, the unphysical mass transfer phenomenon of SCMP pseudopotential LBM that causes the unachievable multi-bubble/droplet configurations is studied. A number of SCMP models involving the typical non-ideal EOSs, forcing schemes, and collision terms of pseudopotential LBM are tested. None of these models are free of the unphysical mass transfer which we call "the big eat the small". The original Shan-Chen MCMP model is also studied, and there is no such unphysical mass transfer phenomenon observed. Through analysing the unphysical mass transfer phenomenon it is found that such problem is most likely caused by the essential attractive interaction forces of pseudopotential LBM. The following conclusions can be drawn within this work:

(1) It is the bubbles or droplets having bigger interface curvature radii that absorb the ones with smaller interface curvature radii, and nothing to do with the volume of bubble/droplet or the area of the interface.

(2) A spurious flow field can be generated between two bubbles or droplets, which is also the flow field of the high-density mass transfer.

(3) The unphysical mass transfer phenomenon is consistent with the attraction force effect.

(4) The unphysical mass transfer can be eliminated using MCMP pseudopotential LBM. 


\section{Acknowledgement}

The authors would like to acknowledge the financial support of this work by the doctoral degree scholarship of China Scholarship Council (CSC) and the University of Nottingham, UK, and this work is also supported by Ningbo Science and Technology Bureau Technology Innovation Team project under Grant No. 2016B10010.

\section{Reference}

[1] Thorne, D. T.; Michael, C. Lattice Boltzmann modeling: an introduction for geoscientists and engineers. 2nd. ed., 2006.

[2] Krüger, T.; Kusumaatmaja, H.; Kuzmin, A.; Shardt, O.; Silva, G.; Viggen, E. M.: The Lattice Boltzmann Method: Principles and Practice; Springer, 2016.

[3] Fei, L.; Luo, K. H. Consistent forcing scheme in the cascaded lattice Boltzmann method. Physical Review E, 2017, 96, 053307.

[4] Gong, W.; Zu, Y.; Chen, S.; Yan, Y. Wetting transition energy curves for a droplet on a square-post patterned surface. Science Bulletin, 2017, 62, 136-142.

[5] Chen, L.; Kang, Q.; Mu, Y.; He, Y.-L.; Tao, W.-Q. A critical review of the pseudopotential multiphase lattice Boltzmann model: Methods and applications. International Journal of Heat and Mass Transfer, 2014, 76, 210-236.

[6] Fei, L.; Luo, K. H.; Lin, C.; Li, Q. Modeling incompressible thermal flows using a central-moments-based lattice Boltzmann method. International Journal of Heat and Mass Transfer, 2018, 120, 624-634.

[7] Li, Q.; Luo, K. H.; Kang, Q. J.; He, Y. L.; Chen, Q.; Liu, Q. Lattice Boltzmann methods for multiphase flow and phase-change heat transfer. Progress in Energy and Combustion Science, 2015, 10.1016/j.pecs.2015.10.001.

[8] Chen, S.; Yan, Y. Y.; Gong, W. A simple lattice Boltzmann model for conjugate heat transfer research. International Journal of Heat and Mass Transfer, 2016, 10.1016/j.ijheatmasstransfer.2016.10.120.

[9] Gong, W.; Yan, Y.; Chen, S.; Giddings, D. Numerical Study of Wetting Transitions on Biomimetic Surfaces Using a Lattice Boltzmann Approach with Large Density Ratio. Journal of Bionic Engineering, 2017, 14, 486-496.

[10] Gunstensen, A. K.; Rothman, D. H.; Zaleski, S.; Zanetti, G. Lattice Boltzmann model of immiscible fluids. Physical Review A, 1991, 43, 4320.

[11] Swift, M. R.; Osborn, W.; Yeomans, J. Lattice Boltzmann simulation of nonideal fluids. Physical Review Letters, 1995, 75, 830.

[12] Inamuro, T.; Ogata, T.; Tajima, S.; Konishi, N. A lattice Boltzmann method for incompressible two-phase flows with large density differences. Journal of Computational Physics, 2004, 198, 628-644.

[13] Yan, Y. Y.; Zu, Y. Q. A lattice Boltzmann method for incompressible twophase flows on partial wetting surface with large density ratio. Journal of Computational Physics, 2007, 227, 763-775.

[14] Shan, X.; Chen, H. Lattice Boltzmann model for simulating flows with multiple phases and components. Physical Review E, 1993, 47, 1815-1819.

[15] Shan, X.; Chen, H. Simulation of nonideal gases and liquid-gas phase transitions by the lattice Boltzmann equation. Physical Review E, 1994, 49, 2941-2948.

[16] Van der Waals, J. D.: Over de Continuiteit van den Gas-en Vloeistoftoestand; Sijthoff, 1873; Vol. 1. 
[17] Carnahan, N. F.; Starling, K. E. Equation of state for nonattracting rigid spheres. The Journal of Chemical Physics, 1969, 51, 635-636.

[18] Yuan, P.; Schaefer, L. Equations of state in a lattice Boltzmann model. Physics of Fluids, 2006, 18, 042101.

[19] He, X.; Doolen, G. D. Thermodynamic foundations of kinetic theory and lattice Boltzmann models for multiphase flows. Journal of Statistical Physics, 2002, 107, 309328.

[20] Yu, Z.; Fan, L. S. Multirelaxation-time interaction-potential-based lattice Boltzmann model for two-phase flow. Physical Review. E: Statistical, Nonlinear, and Soft Matter Physics, 2010, 82, 046708.

[21] Khajepor, S.; Wen, J.; Chen, B. Multipseudopotential interaction: A solution for thermodynamic inconsistency in pseudopotential lattice Boltzmann models. Physical Review E, 2015, 91, 023301.

[22] Xu, A.; Zhao, T. S.; An, L.; Shi, L. A three-dimensional pseudo-potentialbased lattice Boltzmann model for multiphase flows with large density ratio and variable surface tension. International Journal of Heat and Fluid Flow, 2015, 56, 261-271.

[23] Chibbaro, S.; Falcucci, G.; Chiatti, G.; Chen, H.; Shan, X.; Succi, S. Lattice Boltzmann models for nonideal fluids with arrested phase-separation. Physical Review. E: Statistical, Nonlinear, and Soft Matter Physics, 2008, 77, 036705.

[24] Kupershtokh, A.; Medvedev, D.; Karpov, D. On equations of state in a lattice Boltzmann method. Computers \& Mathematics with Applications, 2009, 58, 965-974.

[25] Zhang, C.; Cheng, P. Mesoscale simulations of boiling curves and boiling hysteresis under constant wall temperature and constant heat flux conditions. International Journal of Heat and Mass Transfer, 2017, 110, 319-329.

[26] Li, X.; Cheng, P. Lattice Boltzmann simulations for transition from dropwise to filmwise condensation on hydrophobic surfaces with hydrophilic spots. International Journal of Heat and Mass Transfer, 2017, 110, 710-722.

[27] Li, Q.; Yu, Y.; Zhou, P.; Yan, H. J. Droplet migration on hydrophobichydrophilic hybrid surfaces: a lattice Boltzmann study. RSC Adv., 2017, 7, 14701-14708.

[28] Gong, W.; Chen, S.; Yan, Y. A thermal immiscible multiphase flow simulation by lattice Boltzmann method. International Communications in Heat and Mass Transfer, 2017.

[29] Sehgal, B.; Nourgaliev, R.; Dinh, T. Numerical simulation of droplet deformation and break-up by lattice-Boltzmann method. Progress in Nuclear Energy, 1999, 34, 471-488.

[30] Nekovee, M.; Coveney, P. V.; Chen, H.; Boghosian, B. M. Lattice-Boltzmann model for interacting amphiphilic fluids. Physical Review E, 2000, 62, 8282.

[31] Dörfler, F.; Rauscher, M.; Koplik, J.; Harting, J.; Dietrich, S. Micro- and nanoscale fluid flow on chemical channels. Soft Matter, 2012, 8, 9221.

[32] Gupta, A.; Kumar, R. Lattice Boltzmann simulation to study multiple bubble dynamics. International Journal of Heat and Mass Transfer, 2008, 51, 5192-5203.

[33] Yu, Z.; Yang, H.; Fan, L.-S. Numerical simulation of bubble interactions using an adaptive lattice Boltzmann method. Chemical Engineering Science, 2011, 66, 3441-3451.

[34] Zhang, Q.; Sun, D.; Zhang, Y.; Zhu, M. Lattice Boltzmann modeling of droplet condensation on superhydrophobic nanoarrays. Langmuir, 2014, 30, 12559-12569.

[35] Zhao, H.; Ning, Z.; Kang, Q.; Chen, L.; Zhao, T. Relative permeability of two immiscible fluids flowing through porous media determined by lattice Boltzmann method. International Communications in Heat and Mass Transfer, 2017, 85, 53-61.

[36] Huang, H.; Sukop, M.; Lu, X.: Multiphase lattice Boltzmann methods: Theory and application; John Wiley \& Sons, 2015. 
[37] Gong, S.; Cheng, P. Numerical investigation of droplet motion and coalescence by an improved lattice Boltzmann model for phase transitions and multiphase flows. Computers \& Fluids, 2012, 53, 93-104.

[38] Li, Q.; Luo, K. H.; Li, X. J. Lattice Boltzmann modeling of multiphase flows at large density ratio with an improved pseudopotential model. Physical Review. E:

Statistical, Nonlinear, and Soft Matter Physics, 2013, 87, 053301.

[39] McNaught, A. D.; McNaught, A. D.: Compendium of chemical terminology; Blackwell Science Oxford, 1997; Vol. 1669.

[40] Ratke, L.; Voorhees, P. W.: Growth and coarsening: Ostwald ripening in material processing; Springer Science \& Business Media, 2013. 